\title{
High Serum SI00A 2 Levels Predict Poor Outcome After Acute Primary Intracerebral Hemorrhage
}

\author{
Shen-Zhong Qiu' \\ Guan-Rong Zheng' \\ Cai-Yan $\mathrm{Ma}^{2}$ \\ Bin Chen' \\ Jian-Jun Huang' \\ Ge Huang' \\ Hai Hua' \\ 'Department of Neurosurgery, The First \\ People's Hospital of Fuyang District of \\ Hangzhou City, Hangzhou, People's \\ Republic of China; ${ }^{2}$ Department of \\ Clinical Laboratory, The First People's \\ Hospital of Fuyang District of Hangzhou \\ City, Hangzhou, People's Republic of \\ China
}

\begin{abstract}
Objective: Intracerebral hemorrhage (ICH) triggers an inflammatory cascade that damages brain tissues and worsens functional outcome. S100A12 functions to promote brain inflammation. We aimed to investigate the relationship between serum S100A12 levels and functional outcome in ICH patients.

Methods: Serum S100A12 levels were measured in 101 ICH patients hospitalized within 24 $\mathrm{h}$ after symptom onset. Poor functional outcome was defined as a modified Rankin scale of 3 or greater at 3 months after stroke. Early neurologic deterioration was defined as an increase of $\geq 4$ points in the National Institutes of Health Stroke Scale (NIHSS) score or death at 24 hours from symptoms onset.
\end{abstract}

Results: High serum S100A12 levels were independently correlated with NIHSS score $(\mathrm{t}=$ 5.384, $\mathrm{P}<0.001)$, hematoma volume $(\mathrm{t}=4.221, \mathrm{P}<0.001)$ and serum $\mathrm{C}$-reactive protein levels $(\mathrm{t}=5.068, \mathrm{P}<0.001)$. Serum S100A12 levels were substantially higher in patients with a poor outcome (median, 66.5 versus $37.7 \mathrm{ng} / \mathrm{mL}$; $\mathrm{P}<0.001$ ) or early neurological deterioration (median, 76.5 versus $40.1 \mathrm{ng} / \mathrm{mL}$; $\mathrm{P}<0.001$ ) than in the other remainders, independently predicted a poor outcome (odds ratio, 1.035; 95\% confidence interval, 1.0071.064; $\mathrm{P}=0.015$ ) and early neurologic deterioration (odds ratio, $1.032 ; 95 \%$ confidence interval, 1.003-1.060; $\mathrm{P}=0.027$ ), and significantly discriminated a poor outcome (area under curve, $0.794 ; 95 \%$ confidence interval, $0.702-0.868$ ) and early neurologic deterioration (area under curve, 0.760; 95\% confidence interval, 0.664-0.839) under receiver operating characteristic curve.

Conclusion: High serum S100A12 levels at admission are highly associated with the extent of inflammatory response, severity, a poor functional outcome and early neurologic deterioration in ICH patients, substantializing serum S100A12 as a promising prognostic biomarker for ICH.

Keywords: biomarker, functional outcome, inflammation, intracerebral hemorrhage, early neurological deterioration, S100A12

\section{Introduction}

Intracerebral hemorrhage ( $\mathrm{ICH})$ constitutes $15-20 \%$ of all strokes and is related to high morbidity and mortality. ${ }^{1,2}$ As a common clinical phenomenon, early neurologic deterioration is highly associated with poor outcome after $\mathrm{ICH}^{3}$ During ICH, rapid accumulation of blood within the surrounding brain results in primary injury, subsequently causing secondary brain injury. ${ }^{4}$ Inflammatory response is an important process involved in pathophysiological mechanisms after secondary brain injury relevant to $\mathrm{ICH}^{5}$ Inflammatory cells, including blood-derived leukocytes, microglia/macrophages, astrocytes, and mast cells, and their released cytokines, 
chemokines, prostaglandins, proteases, ferrous iron, and other immunoactive molecules, participate in the whole process of inflammatory reaction after $\mathrm{ICH}^{6}{ }^{6}$ During recent several decades, circulating inflammation-related biomarkers have gained extensive attention for assessing severity as well as predicting prognosis after $\mathrm{ICH}^{7}$

S100A12, a member of the S100 family of calciumbinding proteins, is a ligand for the receptor for advanced glycation end products, toll-like receptor 4, and CD36, which alters inflammation via promoting cellular and immunological pathways. ${ }^{8}$ Clearly, there is a significant up-regulation of S100A12 expression in brain tissues of patients with Alzheimer's disease. ${ }^{9}$ In addition, plasma S100A12 emerged as an independent predictor for poor outcome in patients with acute ischemic stroke. ${ }^{10}$ Also, circulating S100A12 levels have been demonstrated to be tightly associated with inflammation, severity and shortterm mortality in patients with severe traumatic brain injury. ${ }^{11}$ Such data indicate that S100A12, acting as a proinflammatory cytokine, may be a prognostic biomarker for acute brain injury. Interestingly, serum S100A12 appeared to be independently related to 30-day mortality in $\mathrm{ICH}^{12}$ However, circulating S100A12 levels are not investigated regarding its association with 3-month functional outcome and early neurologic deterioration in $\mathrm{ICH}$ patients. In the current study, we enrolled a host of ICH patients, quantified their S100A12 levels in serum and further assessed the relationship between S100A12 levels and 3-month functional outcome in addition to early neurologic deterioration.

\section{Materials and Methods Study Population}

In this prospective, cohort study, were included spontaneous acute ICH patients hospitalized to the First People's Hospital of Fuyang District of Hangzhou City (Hangzhou, China) from January 2017 to January 2020. In the current analysis, we required that patients should be aged $\geq 18$ years, be admitted within $24 \mathrm{~h}$ of stroke onset and not undergo a surgical evacuation of hematoma. We excluded patients with $\mathrm{ICH}$ as a result of secondary causes (eg, brain tumor, vascular malformation, hemorrhagic transformation of cerebral infarction, moyamoya disease and cerebral aneurysm), infection within recent a month, previous neurological diseases (for instance, ischemic stroke, spontaneous subarachnoid hemorrhage, severe traumatic brain injury and $\mathrm{ICH}$ ), use of antiplatelet or anticoagulant medication, autoimmune diseases, malignancies, or severe systemic diseases (for example, uremia, liver cirrhosis, and chronic heart or lung disease). The study was performed in accordance with the tenets of the Declaration of Helsinki and approved by the Human Investigations Committee at the First People's Hospital of Fuyang District of Hangzhou City (No. 2016-04-08). Written informed consent was obtained from legal representatives.

\section{Assessment}

Some routine data were collected, including demographics, vascular risk factors, medication history, medical history, intracerebral hematoma location, and the presence of intraventricular or subarachnoidal extension of hematoma. National Institutes of Health Stroke Scale (NIHSS) was used to assess stroke severity at admission. ${ }^{13}$ Hematoma volume was measured according to $\mathrm{ABC} / 2$ method. ${ }^{14}$ Early neurologic deterioration was defined as an increase of $\geq 4$ points in the NIHSS score or death at 24 hours from symptoms onset. ${ }^{15}$ Participants were followed up until death or completion of 3 months after stroke. Functional outcome was evaluated using the modified Rankin Scale. An unfavorable outcome was defined as a modified Rankin Scale score $>2$ at 3 months. ${ }^{16}$

\section{Immune Analysis}

Peripheral blood samples were collected from ICH patients at admission. The blood samples were immediately centrifuged within $30 \mathrm{~min}$ at $3000 \mathrm{~g}$ for $10 \mathrm{~min}$ and then, serum was preserved at $-80^{\circ} \mathrm{C}$ for subsequent assay. Serum S100A12 levels were quantified in duplicate using a commercially available enzyme-linked immunosorbent assay (Cusabio Biotech Co., Wuhan, Hubei Province, P.R. China). Determinations were completed in batches by the same technician blinded to clinical data.

\section{Statistical Analysis}

Statistical analyses were done using the Statistical Package for the Social Sciences version 19.0 (SPSS Inc., Chicago, IL, USA) and MedCalc 9.6.4.0 (MedCalc Software, Mariakerke, Belgium). Qualitative variables were presented as count (percentage) and were compared for intergroup difference using chi-square test or Fisher exact test as appropriate. Kolmogorov-Smirnov test was performed to determine data distribution of quantitative variables. Data were summarized as median (interquartile range), if non-normally distributed and were presented as mean \pm standard deviation if normally distributed. Intergroup 
comparisons were done using unpaired Student $t$-test or Mann-Whitney $U$-test where appropriate. Comparison of serum S100A12 levels among groups with different modified Rankin Scale scores was performed using the Kruskal-Wallis $H$-test. Bivariate correlations were analyzed using Spearman correlation coefficient. Was configured a multivariate linear logistic regression model, which included variables significantly correlated with serum S100A12 levels in bivariate correlation analysis $(\mathrm{P}<$ 0.05). A binary stepwise logistic regression model, which included significant variables in univariate analysis $(\mathrm{P}<$ $0.05)$, was designed to identify independent variables of an unfavorable outcome or early neurologic deterioration. Odds ratio (OR) and 95\% confidence interval (CI) were reported for showing associations. Area under curve (AUC) and 95\% CI, which was generated using receiver operating characteristic (ROC) curve analysis, were used to assess predictive value. A $P$ value of $<0.05$ was considered significant for other tests, except the KruskalWallis $H$-test, where serum S100A12 levels were compared among 7 groups (ie, modified Rankin Scale score from 0 to 6$)$ and thereby a $\mathrm{P}$ value of $<0.00238(0.05 / 21)$ was considered significant using Bonferroni correction for post hoc test.

\section{Results}

\section{Patients Characteristics}

We initially assessed a total of $148 \mathrm{ICH}$ patients and further excluded 47 patients with intracerebral bleeding as a result of secondary causes (10 cases), infection within recent a month (5 cases), previous neurological diseases ( 8 cases), use of antiplatelet or anticoagulant medication ( 6 cases), autoimmune diseases (4 cases), malignancies (3 cases), severe systemic diseases (9 cases), or loss to follow up (2 cases). Eventually, a total of $101 \mathrm{ICH}$ patients were assessed in the current study.

The stroke patients (58 males and 43 females) had a mean age of $61.2 \pm 13.6$ years (range, 30-89 years). Hypertension and diabetes mellitus were found in 63 $(62.4 \%)$ cases and $14(13.9 \%)$ cases, respectively. Currently, there were 29 (28.7\%) cigarette smokers and $31(30.7 \%)$ alcohol consumers. Patients were hospitalized from 1 to $24 \mathrm{~h}$ (median, $7 \mathrm{~h} ; 25$ th-75th percentiles, 4.5-9.5 h) and their blood samples were acquired from 1.5 to 26.5 h (median, 9 h; lower-upper quartiles, 6.5-13.5 h). Mean systolic arterial pressure and diastolic arterial pressure were $159.9 \pm 25.0 \mathrm{mmHg}$ and $89.6 \pm 18.5 \mathrm{mmHg}$, respectively. There were $23(22.8 \%)$ patients with lobar hemorrhage, $18(17.8 \%)$ with infratentorial hemorrhage and 23 $(22.8 \%)$ with intraventricular hemorrhage. NIHSS score ranged from 1 to (median, 9; 25th -75th, 4-13) and hematoma volume, from 2.0 to $64.8 \mathrm{~mL}$ (median, $14 \mathrm{~mL} ; 25 \mathrm{th}-75 \mathrm{th}, 7.9-21 \mathrm{~mL})$.

\section{Serum SI00A 12 Levels in Relation to Hemorrhagic Severity and Inflammation}

Serum S100A12 levels of ICH patients ranged from 15.6 to $134.3 \mathrm{ng} / \mathrm{mL}$ (median, $44.7 \mathrm{ng} / \mathrm{mL}$; lower-upper quartiles, 35.6-70.7 ng/mL). Table 1 shows that serum S100A12 levels were tightly correlated with diabetes mellitus, NIHSS score, hematoma volume, blood leucocyte count, serum glucose levels and serum C-reactive protein levels. We configured a multivariate linear logistic regression model, which contained the preceding closely correlated variables, and thereby found that serum S100A12 levels were independently correlated with NIHSS score $(\mathrm{t}=5.384, \mathrm{P}<0.001)$, hematoma volume $(\mathrm{t}=4.221$, $\mathrm{P}<0.001)$, and serum $\mathrm{C}$-reactive protein levels $(\mathrm{t}=5.068$, $\mathrm{P}<0.001)$. Their associations are displayed in Figures 1-3.

Table I Correlation Between Serum SI00A I2 Levels and Other Variables Using Spearman Correlation Coefficient in Acute Intracerebral Hemorrhage

\begin{tabular}{|l|c|c|}
\hline Variables & r & P value \\
\hline Age (years) & 0.090 & 0.37 I \\
Gender (male/female) & 0.030 & 0.767 \\
Hypertension & 0.057 & 0.568 \\
Diabetes mellitus & 0.212 & $0.033^{*}$ \\
Current smoking & 0.087 & 0.385 \\
Alcohol consumption & 0.166 & 0.098 \\
Admission time (h) & -0.078 & 0.436 \\
Blood-collection time (h) & -0.035 & 0.729 \\
Systolic arterial pressure (mmHg) & 0.154 & 0.123 \\
Diastolic arterial pressure $(\mathrm{mmHg})$ & 0.148 & 0.140 \\
Lobar hemorrhage & -0.049 & 0.624 \\
Infratentorial hemorrhage & 0.163 & 0.103 \\
Intraventricular hemorrhage & 0.046 & 0.647 \\
NIHSS score & 0.570 & $<0.00 I^{*}$ \\
Hematoma volume (mL) & 0.545 & $<0.00 I^{*}$ \\
Blood leucocyte count $\left(\times 10^{9} / \mathrm{I}\right)$ & 0.344 & $<0.00 I^{*}$ \\
Serum glucose levels $(\mathrm{mmol} / \mathrm{l})$ & 0.326 & $0.00 I^{*}$ \\
Serum CRP levels $(\mathrm{mg} / \mathrm{I})$ & 0.576 & $<0.00 I^{*}$ \\
\hline
\end{tabular}

Notes: NIHSS indicates National Institutes of Health Stroke Scale. CRP denotes $\mathrm{C}$-reactive protein. The asterisk indicates statistical significance $\left({ }^{*} \mathrm{P}<0.05\right)$. 


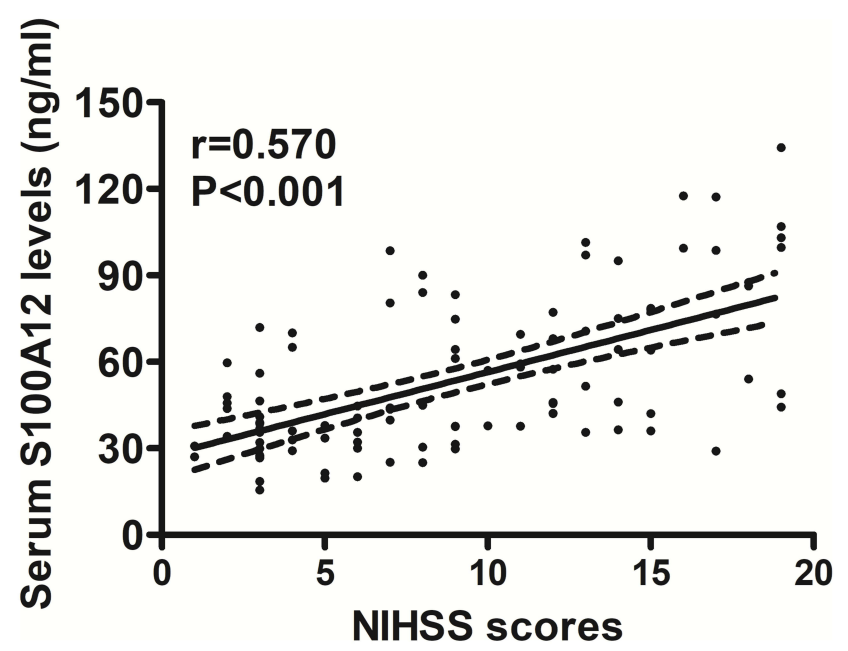

Figure I Correlation of serum SI00AI2 level with National Institutes of Health Stroke Scale scores following acute intracerebral hemorrhage. Bivariate correlation was analyzed using Spearman correlation coefficient and association was reported as $r$ value. NIHSS indicates National Institutes of Health Stroke Scale.

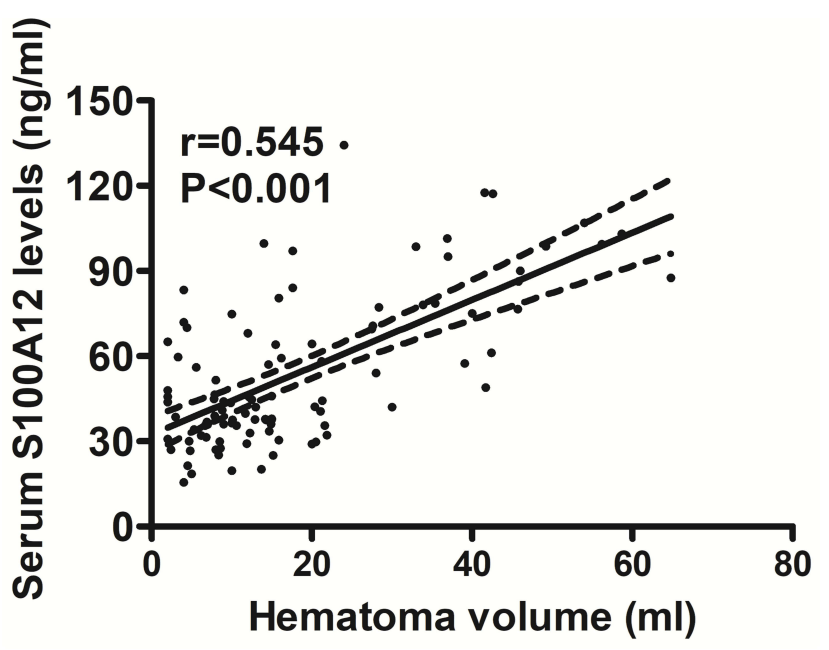

Figure 2 Relationship between serum SI00AI 2 level and hematoma volume after acute intracerebral hemorrhage. Bivariate correlation was analyzed using Spearman correlation coefficient and association was shown as $r$ value.

\section{Prediction of Early Neurologic}

\section{Deterioration}

Among ICH patients, 29 (28.7\%) experienced early neurologic deterioration. ROC curve analysis showed that serum S100A12 levels discriminated patients at risk of early neurologic deterioration with an AUC of 0.760 (95\% CI, 0.664 0.839) (Figure 4). In Table 2, the variables substantially associated with early neurologic deterioration after hemorrhagic stroke were age, NIHSS score, hematoma volume, serum S100A12 levels, blood leucocyte count, serum glucose levels and serum C-reactive protein levels. Using multivariate analysis, NIHSS score, hematoma volume and serum S100A12

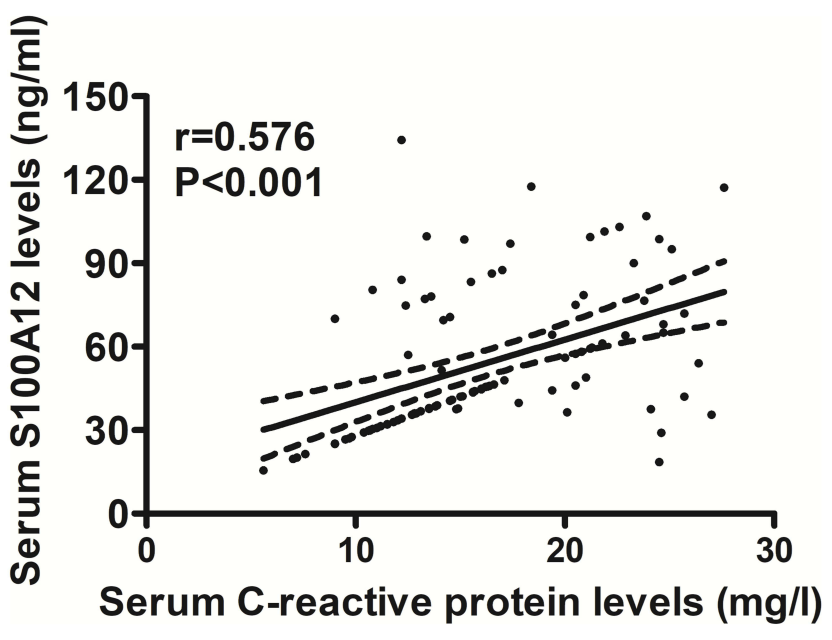

Figure 3 Relation of serum SI00AI2 level to serum C-reactive protein levels after acute intracerebral hemorrhage. Bivariate correlation was analyzed using Spearman correlation coefficient and association was presented as $r$ value.

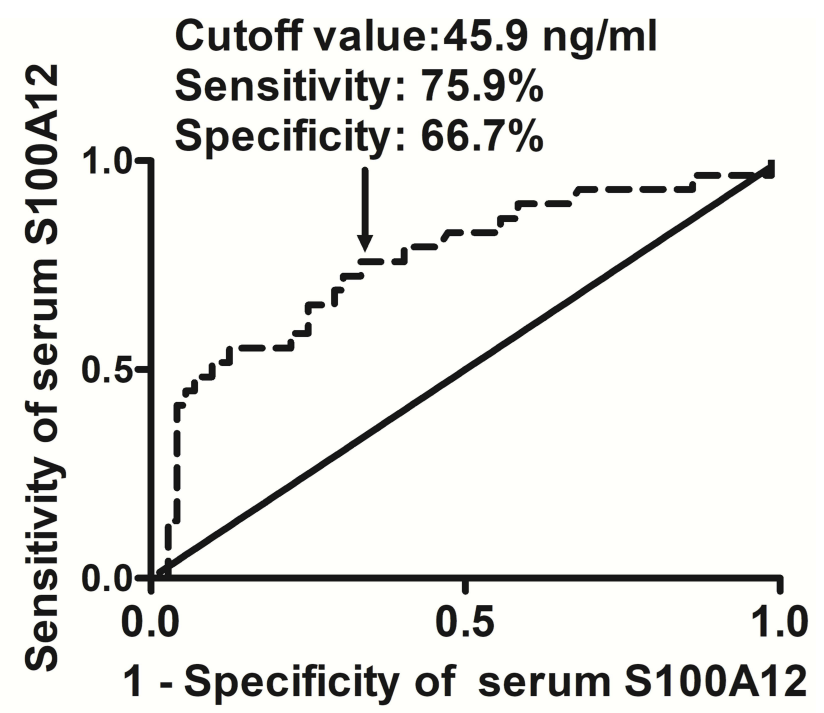

Figure 4 Analysis regarding predictive value of serum SI00AI2 levels for early neurologic deterioration after acute intracerebral hemorrhage. Discriminatory ability was assessed under receiver operating characteristic curve.

levels independently predicted early neurologic deterioration with odds ratio values of $1.206(95 \% \mathrm{CI}, 1.047-1.390 ; \mathrm{P}=$ $0.009), 1.072$ (95\% CI, 1.022-1.124; $\mathrm{P}=0.004)$ and 1.032 (95\% CI, 1.003-1.060; $\mathrm{P}=0.027)$, respectively.

\section{Prediction of 3-Month Unfavorable \\ Outcome}

At 3 months after hemorrhagic stroke, 8, 19, 26, 24, 9, 9 and 6 patients had mRS score $0,1,2,3,4,5$ and 6 , respectively; and thereby, 48 (47.5\%) patients suffered from an unfavorable outcome (modified Rankin scale score above 2). Figure 5 displays that serum S100A12 
Table 2 Factors Associated with Early Neurologic Deterioration in Acute Intracerebral Hemorrhage

\begin{tabular}{|c|c|c|c|}
\hline \multirow[t]{2}{*}{ Variables } & \multicolumn{2}{|c|}{ Early Neurologic Deterioration } & \multirow[t]{2}{*}{$P$ value } \\
\hline & Yes $(n=29)$ & No $(n=72)$ & \\
\hline Age (years) & $68.0 \pm 11.8$ & $58.5 \pm 13.4$ & $0.00 I^{*}$ \\
\hline Gender (male/female) & $15 / 14$ & $43 / 29$ & 0.462 \\
\hline Hypertension & $20(69.0 \%)$ & $43(59.7 \%)$ & 0.386 \\
\hline Diabetes mellitus & $6(20.7 \%)$ & $8(11.1 \%)$ & 0.208 \\
\hline Current smoking & $5(17.2 \%)$ & $24(23.3 \%)$ & 0.107 \\
\hline Alcohol consumption & II (37.9\%) & 20 (27.8\%) & 0.317 \\
\hline Admission time $(\mathrm{h})$ & $6.2(3.5-8.3)$ & $7.5(4.5-10.5)$ & 0.195 \\
\hline Blood-collection time $(\mathrm{h})$ & $8.6(5.6-10.5)$ & $9.3(6.5-14.0)$ & 0.402 \\
\hline Systolic arterial pressure $(\mathrm{mmHg})$ & $165.7 \pm 28.2$ & $157.5 \pm 23.4$ & 0.137 \\
\hline Diastolic arterial pressure $(\mathrm{mmHg})$ & $93.1 \pm 24.4$ & $88.2 \pm 15.5$ & 0.227 \\
\hline Lobar hemorrhage & $6(20.7 \%)$ & $17(23.6 \%)$ & 0.751 \\
\hline Infratentorial hemorrhage & $6(20.7 \%)$ & $12(16.7 \%)$ & 0.633 \\
\hline Intraventricular hemorrhage & $10(34.5 \%)$ & $13(18.1 \%)$ & 0.075 \\
\hline NIHSS score & $15(11-17)$ & $7(3-11)$ & $<0.00$ I* \\
\hline Hematoma volume $(\mathrm{mL})$ & $28.0(\mid 5.5-45.7)$ & $10.3(6.9-15.9)$ & $<0.001 *$ \\
\hline Blood leucocyte count $\left(\times 10^{9} / \mathrm{l}\right)$ & $10.5(7.3-13.8)$ & $8.0(6.5-10.1)$ & $0.022 *$ \\
\hline Serum glucose levels (mmol/l) & $10.5(8.6-11.7)$ & $8.1(7.2-10.2)$ & $0.036 *$ \\
\hline Serum CRP levels (mg/l) & $22.6(20.5-24.5)$ & $13.5(11.1-16.2)$ & $<0.001 *$ \\
\hline Serum SI00AI 2 levels $(\mathrm{ng} / \mathrm{mL})$ & $76.5(46.0-97.0)$ & $40.1(32.2-58.6)$ & $<0.001 *$ \\
\hline
\end{tabular}

Notes: Qualitative variables were presented as count (percentage) and were compared for intergroup difference using chi-square test or Fisher exact test as appropriate. Quantitative variables were summarized as median (interquartile range), if non-normally distributed and were presented as mean \pm standard deviation if normally distributed. Intergroup comparisons were done using unpaired Student $t$-test or Mann-Whitney U-test where appropriate. NIHSS indicates National Institutes of Health Stroke Scale. $\mathrm{CRP}$ denotes $\mathrm{C}$-reactive protein. The asterisk indicates statistical significance $(* \mathrm{P}<0.05)$.

levels were markedly raised, with increasing modified Rankin scale score. In Figure 6, serum S100A12 levels were significantly and positively correlated with modified Rankin scale score. Under ROC curve (Figure 7), serum S100A12 levels significantly distinguished the development of 3-month unfavorable outcome, with AUC at
0.794 (95\% CI, 0.702-0.868). Just as outlined in Table 3, patients with an unfavorable outcome had significantly older age, and higher NIHSS score, hematoma volume, serum S100A12 levels, blood leucocyte count, serum glucose levels and serum C-reactive protein levels, as compared with those with a favorable outcome. Multivariate

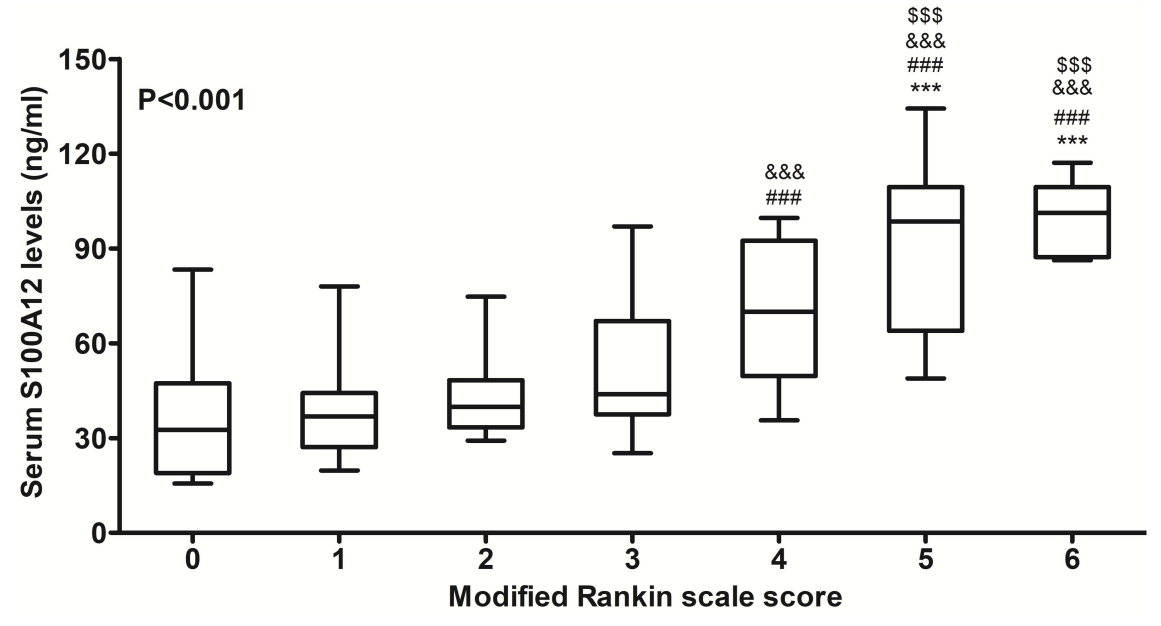

Figure 5 Relationship between serum SI00AI2 levels and modified Rankin scale score among patients with acute intracerebral hemorrhage. Comparison was done using Kruskal-Wallis $\mathrm{H}$-test. In boxplot, ${ }^{* * *} \mathrm{P}<0.00$ I compared with modified Rankin scale score 0 , ${ }^{\# \# ~} \mathrm{P}<0.00 \mathrm{I}$ compared with modified Rankin scale score I, \&\&\& $\mathrm{P}<0.00 \mathrm{I}$ compared with modified Rankin scale score 2 , and ${ }^{\$ \$ \$} \mathrm{P}<0.001$ compared with modified Rankin scale score 3. 


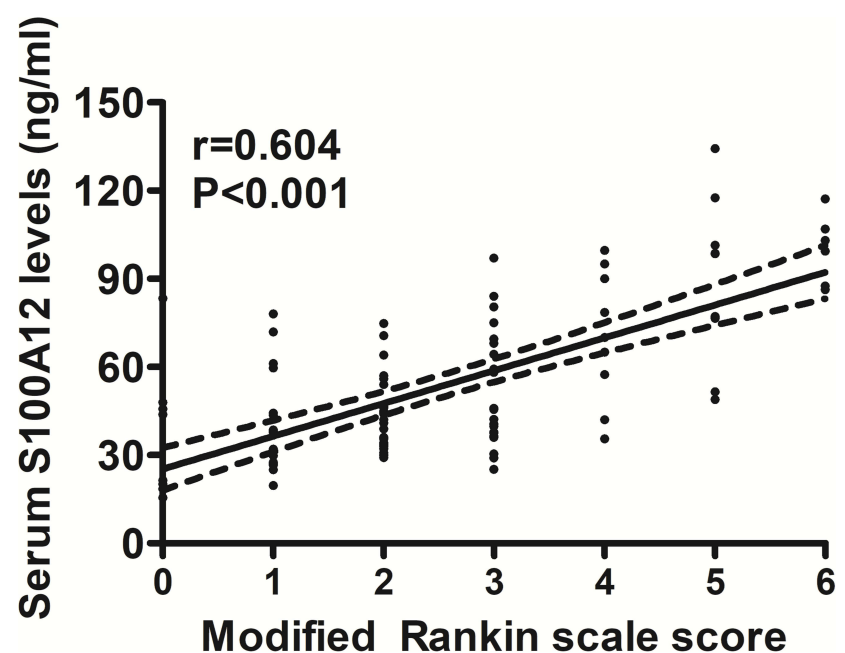

Figure 6 Relationship between serum SI00AI2 level and modified Rankin scale score following acute intracerebral hemorrhage. Bivariate correlation was assessed using Spearman correlation coefficient and association was summarized as $r$ value.

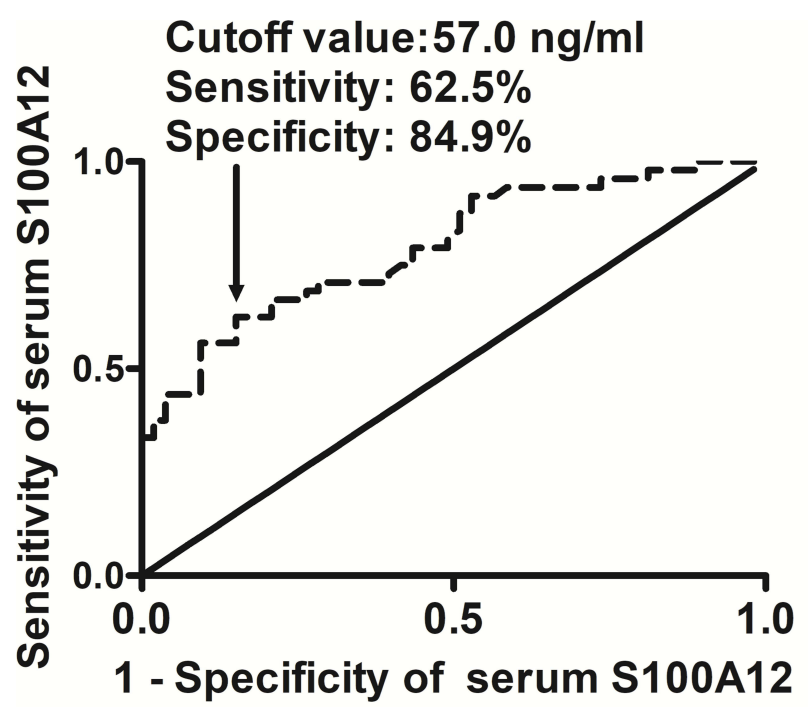

Figure 7 Analysis with respect to predictive ability of serum SI00AI2 levels for 3-month poor outcome after acute intracerebral hemorrhage. Discriminatory capability was evaluated using receiver operating characteristic curve analysis. A poor outcome was referred to as modified Rankin scale score above 2.

analysis showed that NIHSS score (odds ratio, 1.227; 95\% CI, 1.068-1.409; $\mathrm{P}=0.004$ ), hematoma volume (odds ratio, 1.092; 95\% $\mathrm{CI}, 1.029-1.158 ; \mathrm{P}=0.004)$ and serum S100A12 levels (odds ratio, 1.035; 95\% CI, 1.007-1.064; $\mathrm{P}=0.015$ ) emerged as the three independent predictors of 3-month unfavorable outcome after ICH.

\section{Discussion}

The S100 proteins family is identified as a group of calcium-binding proteins encoded by chromosome 1q21 and contains over 20 members with comparable amino acid structures. ${ }^{17}$ These EF-hand proteins predominantly exist in a homodimeric form and, via interacting with several target genes, perform a large range of functions, including regulating cell proliferation and invasion, interacting with the cytoskeleton interplays, and mediating transcription and inflammation. ${ }^{18}$ S100A12, also called calgranulin C, is composed of 92 amino acids and can be secreted and upregulated during acute and chronic infection. ${ }^{19}$ Enough evidence has shown that S100A12 is involved in inflammatory process of numerous diseases, including acute myocardial infarction, ${ }^{20}$ sepsis $^{21}$ and osteoarthritis. ${ }^{22}$ Therefore, upregulated circulating levels of S100A12 are a novel biomarker for many inflammatory diseases, such as inflammatory bowel disease, ${ }^{23}$ dermatomyositisassociated interstitial lung disease ${ }^{24}$ and obstructive sleep apnea. $^{25}$

S100A12 promoted oxygen-glucose deprivation and reperfusion-induced inflammation, oxidative stress and apoptosis in vitro study using PC12 cells. ${ }^{26}$ S100A12 has been found to play an important role in the pathogenesis of diabetic retinopathy by activating retinal microglia via a miR-30a-dependent mechanism. ${ }^{27}$ In a prospective, observational study of 186 patients and 186 controls, a postoperative elevation of serum S100A12 levels was independently correlated with serum C-reactive protein levels after hip fracture surgery in elderly patients. $^{28}$ Another clinical study showed that serum S100A12 levels were substantially higher in patients with severe traumatic brain injury than in healthy controls; and serum concentrations of S100A12 significantly correlated with admission serum concentrations of C-reactive protein, interleukin-6 and tumor necrosis factor-alpha. ${ }^{11}$ In patients with ICH, enhanced serum S100A12 levels were positively correlated with blood glucose concentrations, blood white blood cell count and plasma C-reactive protein concentrations. ${ }^{12}$ In the current study, serum S100A12 levels were independently correlated with serum C-reactive protein levels after acute ICH. Clearly, serum S100A12 may be implicated in inflammation after acute brain injury. Possibly, S100A12 may exert a toxic effect on acute brain injury. However, S100A12 protein has been assumed to be a strong inducer of neurite outgrowth from primary hippocampal neurons. ${ }^{29}$ Thus, its role in acute brain injury remains to be studied in future.

There are two studies demonstrating the relationship between circulating S100A12 levels and severity of acute brain injury. In severe traumatic brain injury, serum 
Table 3 Factors Associated with 3-Month Poor Outcome in Acute Intracerebral Hemorrhage

\begin{tabular}{|c|c|c|c|}
\hline \multirow[t]{2}{*}{ Variables } & \multicolumn{2}{|c|}{ 3-Month Functional Outcome } & \multirow[t]{2}{*}{$P$ value } \\
\hline & mRS 3-6 $(n=48)$ & mRS 0-2 (n= 53) & \\
\hline Age (years) & $65.0 \pm 14.0$ & $57.8 \pm 12.3$ & $0.007^{*}$ \\
\hline Gender (male/female) & $27 / 21$ & $31 / 22$ & 0.820 \\
\hline Hypertension & $32(66.7 \%)$ & 31 (58.5\%) & 0.397 \\
\hline Diabetes mellitus & $9(18.8 \%)$ & $5(9.4 \%)$ & 0.176 \\
\hline Current smoking & 15 (31.3\%) & 14 (26.4\%) & 0.592 \\
\hline Alcohol consumption & 17 (35.4\%) & 14 (26.4\%) & 0.327 \\
\hline Admission time $(\mathrm{h})$ & $6.4(4.5-8.5)$ & $7.5(4.5-12.5)$ & 0.245 \\
\hline Blood-collection time $(\mathrm{h})$ & $8.4(5.5-11.0)$ & $9.6(6.6-14.5)$ & 0.133 \\
\hline Systolic arterial pressure $(\mathrm{mmHg})$ & $162.6 \pm 25.2$ & $157.4 \pm 24.9$ & 0.302 \\
\hline Diastolic arterial pressure $(\mathrm{mmHg})$ & $92.7 \pm 21.9$ & $86.9 \pm 14.5$ & 0.116 \\
\hline Lobar hemorrhage & II (22.9\%) & $12(22.6 \%)$ & 0.974 \\
\hline Infratentorial hemorrhage & II (22.9\%) & 7 (I3.2\%) & 0.203 \\
\hline Intraventricular hemorrhage & $13(27.1 \%)$ & $10(18.9 \%)$ & 0.326 \\
\hline NIHSS score & $12(9-16)$ & $5(3-9)$ & $<0.00$ I* \\
\hline Hematoma volume (mL) & $20.7(14.6-39.5)$ & $8.6(5.0-13.0)$ & $<0.001 *$ \\
\hline Blood leucocyte count $\left(\times 10^{9} / \mathrm{l}\right)$ & $10.1(7.0-12.8)$ & $7.5(6.5-9.5)$ & $0.012 *$ \\
\hline Serum glucose levels (mmol/l) & $9.5(7.5-11.7)$ & $8.1(6.9-10.1)$ & $0.037^{*}$ \\
\hline Serum CRP levels (mg/l) & I8.I (I4.3-22.2) & $13.1(11.0-16.0)$ & $<0.001 *$ \\
\hline Serum SI00AI 2 levels $(\mathrm{ng} / \mathrm{mL})$ & $66.5(41.3-92.5)$ & $37.7(30.0-46.0)$ & $<0.001 *$ \\
\hline
\end{tabular}

Notes: Qualitative variables were presented as count (percentage) and were compared for intergroup difference using chi-square test or Fisher exact test as appropriate. Quantitative variables were summarized as median (interquartile range), if non-normally distributed and were presented as mean \pm standard deviation if normally distributed. Intergroup comparisons were done using unpaired Student $t$-test or Mann-Whitney U-test where appropriate. NIHSS indicates National Institutes of Health Stroke Scale. CRP denotes C-reactive protein. The asterisk indicates statistical significance $(* P<0.05)$.

S100A12 levels were highly correlated with Glasgow coma scale score. ${ }^{11}$ In ICH, serum S100A12 concentrations were positively correlated with NIHSS score and ICH volume using univariate analysis. ${ }^{12}$ In the current study, we used multivariate analysis to verify correlation of serum S100A12 levels with hemorrhagic severity after acute ICH. Serum S100A12 levels were actually independently correlated with NIHSS score and hematoma volume. Overall, our data are supportive of the notion that serum S100A12 levels may be correlated with severity of ICH.

Postoperative serum S100A12 levels were independently associated with the risk of developing postoperative delirium and cognitive dysfunction in a group of elderly patients undergoing hip fracture surgery. ${ }^{28}$ In patients with acute ICH or severe traumatic brain injury, serum S100A12 levels independently predicted 30-mortality and overall survival. ${ }^{11,12}$ In particular, plasma S100A12 emerged as an independent predictor for a poor functional outcome after acute ischemic stroke. ${ }^{10}$ However, no studies have reported association of serum S100A12 levels with early neurologic deterioration and functional outcome after ICH. We defined modified Rankin scale score above
2 as a poor outcome and found that serum S100A12 levels, NIHSS score and hematoma volume were the three independent predictors for 3-month poor functional outcome and early neurologic deterioration. Moreover, serum S100A12 levels exhibit an efficient discriminatory capability for a poor clinical outcome and early neurologic deterioration under ROC curve. In conclusion, serum S100A12 may serve as a useful prognostic biomarker for $\mathrm{ICH}$.

$\mathrm{ICH}$ is a cerebrovascular disease relevant to systemic inflammation and neuroinflammation. ${ }^{30}$ Circulating C-reactive protein levels can reflect extent of systemic inflammation. $^{31}$ Interestingly, it has been found that C-reactive protein in peripheral blood of $\mathrm{ICH}$ patients is at least partly originated from activated neuroglial cells. ${ }^{32}$ Thus, circulating C-reactive protein emerges as an inflammatory prognostic biomarker of $\mathrm{ICH}^{3}{ }^{33}$ Clearly, S100A12, as an inflammatory protein, can be released from peripheral cells after inflammatory stimulation. ${ }^{8}$ Intriguingly, S100A12 is also generated from neuroglial cells and its obvious up-regulation in brain tissue has been found after acute brain injury. $^{26}$ In the current study, serum S100A12 levels were intimately related to serum C-reactive protein levels. Hence, it is assumed that serum S100A12 may be an 
inflammatory biomarker. Alternatively, in this study, serum S100A12 levels were highly correlated with hemorrhagic severity assessed by NIHSS score and hematoma volume, indicating that serum S100A12 may be at least partly derived from central nervous system. In a word, it is hypothesized that serum S100A12 may be an inflammatory prognostic biomarker.

There are two limitations in our study. First, a P value $<$ 0.05 was considered statistically significant for nearly all tests in the current study, and some associations in this study have significant differences of $\mathrm{P}<0.05$, but not less than 0.01. Thereby, statistical significance may not be enough big. Second, in order to decrease confounding effects of some therapies on functional recovery of patients with $\mathrm{ICH}$, patients undergoing a surgical evacuation of hematoma have been excluded in the current study. However, other therapies in the acute phase, such as mannitol, albumin and antibiotic injections, may affect functional recovery of $\mathrm{ICH}$ patients. So, potential bias may have been induced. Overall, this study is preliminary and is just considered as a pilot one. Consequently, a larger cohort of patients are warranted to validate the close relationship between serum S100A12 levels and severity in addition to prognosis after acute ICH.

\section{Conclusions}

This study, for the first time, used multivariate analysis to discern the relationship between serum S100A12 levels and hemorrhagic severity, inflammation, early neurologic deterioration in addition to long-term functional outcome. In the current study, high serum S100A12 levels were independently associated with NIHSS score, hematoma volume, serum C-reactive protein levels, early neurologic deterioration and 3-month poor outcome, indicating that high serum S100A12 levels may be highly correlated with inflammatory response, severity and long-term functional outcome after ICH. Hence, serum S100A12 may be a potential inflammatory biomarker for predicting functional outcome and assessing severity after acute ICH.

\section{Abbreviations}

AUC, area under curve; CI, confidence interval; CT, computerized tomography; ICH, intracerebral hemorrhage; NIHSS, National Institutes of Health Stroke Scale; OR, odds ratio; ROC, receiver operating characteristic.

\section{Acknowledgments}

This work is financially supported by Zhejiang Provincial Medical and Health Technology Project (grant.2019KY554).

\section{Disclosure}

The authors declare that there were no conflicts of interest.

\section{References}

1. Chan S, Hemphill JC. Critical care management of intracerebral hemorrhage. Crit Care Clin. 2014;30(4):699-717. doi:10.1016/j. ccc.2014.06.003.

2. Katsuki M, Kakizawa Y, Nishikawa A, Yamamoto Y, Uchiyama T. Endoscopic hematoma removal of supratentorial intracerebral hemorrhage under local anesthesia reduces operative time compared to craniotomy. Sci Rep. 2020;10(1):10389. doi:10.1038/s41598-02067456-x.

3. Leira R, Dávalos A, Silva Y, et al. Early neurologic deterioration in intracerebral hemorrhage: predictors and associated factors. Neurology. 2004;63(3):461-467. doi:10.1212/01.WNL.0000133204. 81153.AC

4. Provencio JJ, Da silva IR, Manno EM. Intracerebral hemorrhage: new challenges and steps forward. Neurosurg Clin N Am. 2013;24 (3):349-359. doi:10.1016/j.nec.2013.03.002

5. Hostettler IC, Seiffge DJ, Werring DJ. Intracerebral hemorrhage: an update on diagnosis and treatment. Expert Rev Neurother. 2019;19 (7):679-694. doi:10.1080/14737175.2019.1623671.

6. Shi K, Tian DC, Li ZG, Ducruet AF, Lawton MT, Shi FD. Global brain inflammation in stroke. Lancet Neurol. 2019;18 (11):1058-1066. doi:10.1016/S1474-4422(19)30078-X.

7. Zhang Z, Zhang Z, Lu H, Yang Q, Wu H, Wang J. Microglial polarization and inflammatory mediators after intracerebral hemorrhage. Mol Neurobiol. 2017;54(3):1874-1886. doi:10.1007/ s12035-016-9785-6.

8. Pietzsch J, Hoppmann S. Human S100A12: a novel key player in inflammation? Amino Acids. 2009;36(3):381-389. doi:10.1007/ s00726-008-0097-7.

9. Shepherd CE, Goyette J, Utter V, et al. Inflammatory S100A9 and S100A12 proteins in Alzheimer's disease. Neurobiol Aging. 2006;27 (11):1554-1563. doi:10.1016/j.neurobiolaging.2005.09.033.

10. Wakisaka Y, Ago T, Kamouchi M, et al. Plasma S100A12 is associated with functional outcome after ischemic stroke: research for biomarkers in ischemic stroke. $J$ Neurol Sci. 2014;340(1-2):75-79. doi:10.1016/j.jns.2014.02.031.

11. Feng MJ, Ning WB, Wang W, et al. Serum S100A12 as a prognostic biomarker of severe traumatic brain injury. Clin Chim Acta. 2018;480:84-91. doi:10.1016/j.cca.2018.01.044.

12. Qian SQ, He SR, Li BB, Qian J, Zheng XD. Serum S100A12 and 30-day mortality after acute intracerebral hemorrhage. Clin Chim Acta. 2018;477:1-6. doi:10.1016/j.cca.2017.11.032.

13. Kasner SE. Clinical interpretation and use of stroke scales. Lancet Neurol. 2006;5(7):603-612. doi:10.1016/S1474-4422(06)70495-1.

14. Kothari RU, Brott T, Broderick JP, et al. The ABCs of measuring intracerebral hemorrhage volumes. Stroke. 1996;27(8):1304-1395. doi:10.1161/01.str.27.8.1304.

15. Godoy DA, Boccio A. Early neurologic deterioration in intracerebral hemorrhage: predictors and associated factors. Neurology. 2005;64 (5):931-932. doi:10.1212/wnl.64.5.931-a.

16. Schneider H, Huynh TJ, Demchuk AM, et al. Combining spot sign and intracerebral hemorrhage score to estimate functional outcome: analysis from the PREDICT cohort. Stroke. 2018;49(6):1511-1514. doi:10.1161/STROKEAHA.118.020679.

17. Donato R, Cannon BR, Sorci G, et al. Functions of S100 proteins. Curr Mol Med. 2013;13(1):24-57. doi:10.2174/1566524138044 86214

18. Schäfer BW, Heizmann $\mathrm{CW}$. The S100 family of EF-hand calcium-binding proteins: functions and pathology. Trends Biochem Sci. 1996;21(1):134-140. doi:10.1016/s0968-0004(96)80167-8. 
19. Goyette J, Geczy CL. Inflammation-associated S100 proteins: new mechanisms that regulate function. Amino Acids. 2011;41(4):821-842.

20. Grauen Larsen H, Yndigegn T, Marinkovic G, et al. The soluble receptor for advanced glycation end-products (sRAGE) has a dual phase-dependent association with residual cardiovascular risk after an acute coronary event. Atherosclerosis. 2019;287:16-23. doi:10.1016/ j.atherosclerosis.2019.05.020

21. Zhang Z, Han N, Shen Y. S100A12 promotes inflammation and cell apoptosis in sepsis-induced ARDS via activation of NLRP3 inflammasome signaling. Mol Immunol. 2020;122:38-48. doi:10.1016/j. molimm.2020.03.022.

22. Nakashima M, Sakai T, Hiraiwa H, et al. Role of S100A12 in the pathogenesis of osteoarthritis. Biochem Biophys Res Commun. 2012;422(3):508-514. doi:10.1016/j.bbrc.2012.05.036.

23. van de Logt F, Day AS. S100A12: a noninvasive marker of inflammation in inflammatory bowel disease. J Dig Dis. 2013;14(2):62-67. doi:10.1111/1751-2980.12012

24. Lou Y, Zheng Y, Fan B, et al. Serum S100A12 levels are correlated with clinical severity in patients with dermatomyositis-associated interstitial lung disease. J Int Med Res. 2020;48(4):30006051 9887841. doi:10.1177/0300060519887841

25. Demirci Sağlam G, Zamani A, Yosunkaya Ş, Kilinc I. Serum S100A12 and S100B proteins are independent predictors of the presence and severity of obstructive sleep apnea. Turk J Med Sci. 2019;49(3):746-754. doi:10.3906/sag-1806-147.

26. Zhang X, Shen R, Shu Z, Zhang Q, Chen ZS. S100A12 promotes inflammation and apoptosis in ischemia/reperfusion injury via ERK signaling in vitro study using PC12 cells. Pathol Int. 2020;70 (7):403-412. doi:10.1111/pin.12924.
27. Dong N, Wang Y. MiR-30a Regulates S100A12-induced retinal microglial activation and inflammation by targeting NLRP3. Curr Eye Res. 2019;44(11):1236-1243. doi:10.1080/02713683.2019.16 32350 .

28. Li QH, Yu L, Yu ZW, et al. Relation of postoperative serum S100A12 levels to delirium and cognitive dysfunction occurring after hip fracture surgery in elderly patients. Brain Behav. 2019;9(1):e01176. doi:10.1002/brb3.1176

29. Mikkelsen SE, Novitskaya V, Kriajevska M, et al. S100A12 protein is a strong inducer of neurite outgrowth from primary hippocampal neurons. J Neurochem. 2001;79(4):767-776. doi:10.1046/j.14714159.2001.00605.x

30. Fonseca S, Costa F, Seabra M, et al. Systemic inflammation status at admission affects the outcome of intracerebral hemorrhage by increasing perihematomal edema but not the hematoma growth. Acta Neurol Belg. 2021;121(3):649-659. doi:10.1007/s13760-01901269-2.

31. Sproston NR, Ashworth JJ. Role of C-reactive protein at sites of inflammation and infection. Front Immunol. 2018;9:754. doi:10. 3389/fimmu.2018.00754.

32. Di Napoli M, Godoy DA, Campi V, et al. C-reactive protein in intracerebral hemorrhage: time course, tissue localization, and prognosis. Neurology. 2012;79(7):690-699. doi:10.1212/WNL.0b $013 \mathrm{e} 318264 \mathrm{e} 3$ be

33. Wang J, Wang W, Liu Y, Zhao X. Associations between levels of high-sensitivity C-reactive protein and outcome after intracerebral hemorrhage. Front Neurol. 2020;11:535068. doi:10.3389/fneur.20 20.535068 .
Neuropsychiatric Disease and Treatment

\section{Publish your work in this journal}

Neuropsychiatric Disease and Treatment is an international, peerreviewed journal of clinical therapeutics and pharmacology focusing on concise rapid reporting of clinical or pre-clinical studies on a range of neuropsychiatric and neurological disorders. This journal is indexed on PubMed Central, the 'PsycINFO' database and CAS, and is the official journal of The International Neuropsychiatric Association (INA). The manuscript management system is completely online and includes a very quick and fair peer-review system, which is all easy to use. Visit http://www.dovepress.com/testimonials.php to read real quotes from published authors. 